\title{
Fables et métaphores comme arguments logiques du discours politique dans la Rhétorique d'Aristote
}

\author{
JEAN-LOUIS LABARRIERE \\ Centre Louis Gernet \\ CNRS/EHESS, Paris
}

\begin{abstract}
RESUMO: Aristóteles considera que, no âmbito das provas morais, a prova de caráter é a mais adequada ao discurso político, enquanto a que procura excitar as paixões dos ouvintes é mais adequada ao discurso judiciário. Esta prova (pistis), que tem o objetivo de fazer com que o orador pareça ter uma certa qualidade, torna a arenga mais nobre que o discurso de defesa. No que concerne às provas lógicas, o discurso de defesa tem vantagem pelo fato de que, retomando 0 passado, o orador pode valer-se de entimemas. A arenga, voltada para o futuro, usa exemplos (paradeigmata) que são de dois tipos: exemplos históricos, que deveriam ser os mais fortes, ou fábulas e parábolas às quais o orador recorre, uma vez que a história é freqüentemente muda. Essa utilização de fábulas é questionada no presente ensaio: não se deve utilizá-las a menos que nelas se possa encontrar semelhanças, traço que caracteriza quem sabe construir metáforas.
\end{abstract}

PALAVRAS-CHAVE: Aristóteles; retórica; discurso político; provas; metáforas.

Introduire à l'usage des fables et métaphores comme arguments logiques dans le discours politique implique tout d'abord de procéder à deux rappels préalables:

1) Dans sa Rhétorique (Rhét.), notamment en I, 3, Aristote distingue trois genres ou espèces (eidê) de discours en fonction des trois seules sortes possibles d'auditeurs: l'auditeur est soit "spectateur" (theôron), soit "juge" (kritês), et en ce dernier cas il juge soit du passé, comme les juges ou jurés d'un tribunal, soit de l'avenir, comme les membres de l'assemblée du peuple. On appellera donc "épidictique", terme qu'on pourrait traduire par "apologétique" ou "panégyrique", le discours dont l'auditeur est spectateur et se prononce principalement sur le talent de l' orateur vantant les mérites de X ou Y, tandis qu'est "judiciaire" (dikanikon) le discours s'adressant à un auditeur en position de juge dans un tribunal. Quant au discours s'adressant aux membres de l'assemblée du peuple, la harangue, Aristote le qualifie de délibératif (sumbouleutikon) et estime que seul l'auditeur des débats politiques est "juge" à proprement parler (Rhét., II, 18, 1391b7-21) parce qu'il est le seul à avoir à distinguer entre des opinions portant sur des sujets qui sont authentiquement matière à délibération et qui de 

dans la Rhétorique d'Aristote.

surcroît lui sont propres car communs à tous les citoyens, ce que ne sont pas les sujets du discours judiciaire ou plaidoyer, lequel porte, par définition, sur des sujets "étrangers" au destinataire du discours. C'est d'ailleurs ce qui permet à Aristote de soutenir que "la pratique de la harangue est moralement plus belle et plus politique que celle des discours relatifs aux contrats" (Rhét., I, 1, 1354b24) du fait qu'il y a moins de profit à rechercher en captant l'attention de l'auditeur par des procédés destinés à lui faire oublier le sujet du discours: à l'inverse, l'auditeur du discours politique est toujours prompt à ramener à une cause qui est sienne.

Ajoutons encore quelques précisions au sujet du discours délibératif ou politique puisque c'est le seul dont je m'occuperai ici. Qui prononce un tel discours se trouve dans la position de conseiller ou déconseiller sur ce qu'il estime être utile ou nuisible pour l'avenir de la cité. Il devra donc se munir des prémisses propres au genre de discours qu'il aura à prononcer, lequel porte principalement sur les revenus, la guerre et la paix, les importations et les exportations, la protection du territoire et enfin la législation (Rhét., I, 4, 1359b191360a37). Cette connaissance des dossiers, il pourra l'acquérir en lisant des traités de politique afin de n'être point creux, mais il ne devra la manier qu'avec la plus extrême prudence afin de ne pas entrer dans des compétences scientifiques et techniques qui excéderaient les capacités de ses auditeurs. En effet, le membre de l'assemblée du peuple est un homme "simple", pour ne pas dire inculte, comme le souligne régulièrement Aristote'. Il ne faut donc pas confondre son discours avec un exposé mathématique, ni tirer ses arguments de trop loin. Ce qui me conduit à mon second rappel préalable.

2) Posant que "le convaincant est convaincant pour quelqu'un" (Rhét, I, 2, 1356b28), Aristote distingue différents types de "preuves", pisteis, lesquelles sont plutôt des "moyens de convaincre" que des preuves à proprement parler, et certainement pas des preuves au sens moderne du terme. La première grande distinction consiste à distinguer les preuves "atechniques" des preuves "en-techniques" (Rhét., I, 2, 1355a35-39). Cette première distinction peut être considérée comme inverse de celle que nous serions tentés de faire aujourd'hui: Aristote appelle a-techniques ou non-techniques les preuves qui ne résultent pas de notre discours mais sont préalablement données, comme les témoignages, les aveux sous la torture, les écrits, etc., bref ce que nous appellerions sans doute aujourd'hui des preuves "matérielles", lesquelles appartiennent en priorité, dit-il, au genre judiciaire (Rhét., I, 15, 1357a22). Sont en revanche considérées comme (en)techniques, les "preuves" qui relèvent de notre propre discours et ne doivent leur force de persuasion qu'à notre discours, et non à un quelconque préjugé favorable à notre égard que pourrait avoir nos auditeurs. C'est essentiellement de ces "preuves techniques" que s'occupe la Rhétorique. Où l'on voit clairement que ces fameuses pisteis n'ont rien à voir avec des preuves au sens policier du terme.

Maintenant, ces preuves techniques fournies par le discours lui-même se divisent elles-mêmes en trois espèces:

i) les arguments démonstratifs eux-mêmes, à commencer par les fameux enthymèmes, pendants rhétoriques des syllogismes dialectiques, et les paradigmes ou exemples, pendants rhétoriques des inductions dialectiques,

ii) les preuves manifestant le caractère de l'orateur,

iii) les preuves exploitant les passions des auditeurs. 
C'est ce que la tradition a codifié en "preuves logiques et objectives" d'un côté et en "preuves morales et subjectives" de l'autre, c'est-à-dire en "convaincre", fidem facere, et en "émouvoir", animos impellere.

Si chaque genre oratoire doit bien entendu employer des arguments démonstratifs, il faut relever, trait essentiel pour mon propos, que la déduction est plus à sa place dans le discours judiciaire du fait même qu'il porte sur le passé, tandis que l'induction sera plus à sa place dans le discours délibératif parce qu'il porte sur l'avenir. Et il faut encore relever, trait non moins essentiel, que la preuve morale et subjective la plus adaptée au plaidoyer est celle qui cherche à exploiter les passions des auditeurs, tandis que celle qui le sera le plus à la harangue est celle qui repose sur le caractère manifesté par l'orateur à la seule aide de son discours. D'où ce singulier croisement paradoxal des preuves entre le discours judiciaire et le discours délibératif: le plaidoyer a pour lui la plus forte des preuves logiques, mais contre lui la plus répréhensible des preuves morales; la harangue a pour elle la plus forte et la plus noble des preuves morales, celle qu'Aristote considère comme la maîtresse preuve (kuriôtaton en Rhét., I, 1, 1355a7 et kuriôtatên en I, 2, 1356a13), à savoir la preuve fondée sur le caractère de l'orateur, mais elle est desservie par la plus grande faiblesse de son argument logique. C'est ce qui explique qu'Aristote puisse écrire: “il convient mieux à l'honnête homme (tôi epieikei) de paraître vertueux (khrêston phainesthai) que de présenter un discours exact" (Rhét., III, 17, 1418a40-41).

Ce qu'il me faut maintenant expliquer et qui me conduira au cœur de mon argument.

\section{Du paradigme de l'homme de qualité à la métaphore}

Pour que la preuve par le caractère soit efficace, il est nécessaire, dit Aristote, que "l'orateur paraisse d'une certaine qualité (to poion tina phainesthai)" (Rhét., II, 1, 1377b22, 26, 29). Il faut faire "supposer" (hupolambanein, 1377b27) aux auditeurs que l'on est dans une certaine disposition à leur égard et faire en sorte qu'ils le soient aussi vis-à-vis de vous. Voilà qui n'est possible, soutient Aristote, qu'en faisant paraître sa prudence, sa vertu et sa bienveillance (phronêsis kai aretê kai eunoia, 1378a8). Or, si les moyens de paraître amical et bienveillant se tirent de ce qui est dit des passions (II, 4, pour la philia et sans doute II, 7, pour l'eunoia, bien qu'il y s'agisse à vrai dire de la kharis), ceux de parâtre prudent et vertueux, c'est-à-dire équitable (epieikês et spoudaios semblent synonymes en 1378a12 et 16) se tirent quant à eux de l'exposé relatif aux vertus, soit du chapitre consacré au genre épidictique, c'est-à-dire "panégyrique" ou "apologétique" (I, 9). Aristote soutient en effet que les moyens destinés à se présenter doté de telle ou telle qualité sont les mêmes que ceux que l'on emploie pour représenter autrui sous tel ou tel jour. Se devant avant tout de "paraître vertueux", l'orateur politique puisera donc aux mêmes sources que le panégyriste en cherchant d'abord ce qui peut être loué. C'est-à-dire aux actions accomplies consécutivement à une proairesis, à un "bon choix": tel est, en effet, le propre de l'homme vertueux, équitable (I, 9, 1367b21-23). Ainsi paraîtra-t-on digne de confiance. Nous pouvons ainsi transposer à la preuve par le caractère ce qu'Aristote dit ailleurs de la narration, diêgêsis, qu'il faut aussi rendre éthique, ce qui n'est possible que "si nous voyons (eidômen) ce qui fait le caractère éthique” (III, 16, 1417a17). C'est pourquoi, même si malheureusement pour moi en raison 

dans la Rhétorique d'Aristote.

de l'objet de ce colloque ! la narration n'a pas sa place dans les harangues du fait qu'elle n'a rien à voir avec la nature du conseil, rien n'empêche pourtant d'en récupérer ce qui y est dit de la façon de faire paraître "éthique" son discours. Il faut, dit Aristote, manifester sa proairesis, son choix ou intention "non comme si l' on parlait en fonction d'un calcul, comme on le fait maintenant, mais comme si c'était en fonction de sa préférence" (III, 16, 1417a2425). Autrement dit, les intentions ne doivent pas paraître procéder d'un calcul, mais de la noblesse d'âme, et cela par la seule force du discours puisqu'il ne s'agit pas de paraître vertueux en raison d'un préjugé favorable des auditeurs à votre égard. Comme le dit encore Aristote: "introduis-toi immédiatement avec telle qualité déterminée, afin qu'ils te voient comme tel, et même l'adversaire; mais fais-le en le dissimulant (lanthanôn)" (1417b7-8).

L'explication de ce dernier point - tout l'art de l'orateur consiste à masquer ses effets passe en premier lieu par la prise en considération de l'argument "logique" propre au discours délibératif. J'ai rappelé précédemment que le paradeigma, le "paradigme" ou "exemple", était le pendant, antistrophos, rhétorique de l'induction dialectique et que ce type d'argument était particulièrement approprié à la harangue ou discours politique du fait que son objet est de conseiller sur ce qu'on estime être bon pour l'avenir de la cité. Il me faut maintenant préciser que le paradeigma n'est pas le pendant rhétorique de toute forme d'induction, mais seulement de celle qui va "de la partie à la partie, du semblable au semblable, lorsque les deux termes tombent sous le même genre, mais que l'un est plus connu que l'autre" (Rhét., I, 2, 1357b28-30). L'induction rhétorique ne saurait donc être mise en forme de syllogisme, ainsi qu'il en va de l'induction parfaite qui, dit Aristote, conclut en considérant tous les cas (Premiers Analytiques, II, 23, 68b28-35), chose impossible dans un discours car cela demande trop d'exemples et qu'une telle multiplication lasserait l'auditeur (Rhét., II, 20, 1394a11-16). Les considérations pragmatiques pèsent ici de tout leur poids: il faut être bref, ce qui ne signifie pas être incomplet, mais qui implique de condenser son argumentation en omettant ce qui serait logiquement nécessaire, mais lasserait le public. La même raison conduit Aristote à préciser que si d'aventure l'orateur politique pouvait disposer d'enthymèmes, il devrait toujours préférer l'enthymème réfutatif (elegtikos) au démonstratif (deiktikos). En effet, puisque toujours on conteste nécessairement quelque thèse ou que l'on s'en prend à un adversaire (Rhét., II, 18, 1391b12-14), c'est grâce à un bref rapprochement des contraires que les choses apparaîtront plus manifestes aux auditeurs (Rhét., II, 23,1400b29-32). Et Aristote de préciser que les enthymèmes réfutatifs les plus prisés sont soit ceux dont on peut prévoir le déroulement dès le début, car l'auditeur s'en sent réhaussé, soit ceux que l'on comprend juste après coup au fur et à mesure de leur énonciation (Rhét., II, 23, 1400b32-35; III, 10, 1410b20-27). Bref, il ne faut pas perdre son auditeur, mais l'emmener avec soi, sans pour autant qu'il s'en aperçoive.

Comment parvenir à cela en maniant des paradigmes et en usant de la preuve par le caractère? En prenant tout d'abord en considération ce fait qu'il y a deux espèces de paradigmes: ceux tirés des faits historiques passés et ceux inventés par l'orateur lui-même. L'exemple même du paradigme tiré des faits historiques est celui de la garde demandée par les futurs tyrans - puisque Pisistrate a demandé une garde avant et afin de s'emparer du pouvoir, alors si Denys en demande une aujourd'hui, c'est qu'il s'apprête à devenir tyran (Rhét., I, 2, 1357b26-36). Quant aux paradigmes inventés par l'orateur, paradigmes qui comme tout paradigme sont, je le rappelle, des arguments "logiques", ils se subdivisent 
eux-mêmes en deux espèces (Rhét., II, 20, 1393a28-94a2). Voilà qui explique mon titre puisque nous avons:

1) les paraboles, dont les discours de Socrate comparant le tirage au sort des magistrats à la désignation par le sort d'un pilote de navire constituent l'archétype,

2) les fables, ésopiques ou lybiennes, comme lorsque Stésichore conta un apologue aux citoyens d'Himère destiné à les dissuader de donner une garde au stratège Phalaris (1394b8-22).

En droit, les paradigmes tirés de faits historiques devraient avoir la précellence car ils sont plus utiles pour la délibération du fait qu'ils portent sur des événements réels et que bien souvent l'avenir ressemble au passé, mais comme l'histoire est souvent muette et que les situations peuvent être inédites, l'orateur aura de fait plus souvent recours aux fables et paraboles, estimées d'ailleurs par Aristote plus faciles à se procurer que les exemples historiques (1394a2-8). Mais cette facilité ne doit pas faire illusion: pour pouvoir inventer des fables ou des paraboles, il faut impérativement savoir "voir le semblable" (to homoin horan), tâche à laquelle la philosophie permet de s'entraîner (voir aussi Rhét., III, 9, 1404a1516). Ainsi, en guise d'induction reposant sur le passage d'un cas semblable à un autre cas semblable, l'orateur politique aura le plus souvent recours à du semblable inventé et narré. Cette faculté d'apercevoir les ressemblances, faculté sans laquelle il est vain de chercher à inventer fables et paraboles, c'est ce que la Poétique nomme "bien métaphoriser" (eu metapherein, Poét., 22, 1459b6-8). Que dans la Poétique Aristote tienne cette qualité pour un don naturel, car ce serait la seule chose qu' on ne puisse prendre à autrui, ne doit pas nous arrêter: dans la perspective qui en ce passage de la Rhétorique est la sienne, Aristote traite de cette faculté comme d'une question technique, ce qui implique qu'on puisse l'acquérir.

Concluons ce point en notant que la preuve logique la plus appropriée à la harangue s'ouvre à la question du style, lexis, et de la métaphore d'une façon que nous ne pouvons tenir pour superflue, comme si la question du style et le maniement des métaphores étaient étrangères à l'argumentation elle-même. Ce qu'il me faut maintenant expliquer et qui nous ramènera, via les qualités de la métaphore, à cet art de la dissimulation par lequel l'orateur politique paraîtra vertueux.

\section{Des qualités de la métaphore au style qui convient à un homme de qualité}

En ouvrant ce qu'il est convenu d'appeler Peri lexeôs, "Traité du style" (Rhét., III, 112), Aristote écrit que le style, lexis, "contribue pour beaucoup à ce que le discours paraisse d'une certaine qualité (to phainênai poion tina ton logon)" (III, 1, 1403b17-18). Bien plus, il faudra encore le prononcer comme il faut, et tel sera le rôle de l'hupokrisis, notion qu'il vaudrait mieux traduire, si l'on suit les canons de la rhétorique romaine, par pronunciatio que par actio, car il est chez Aristote plus question de "mise en scène de la voix" que de gestes ou de pantomime. Pourquoi cela? Parce qu'en fonction même de l'auditoire à qui l'on s'adresse, nombreux et inculte en ce qui concerne l'auditoire de l'orateur politique, il faut chercher à le convaincre par des moyens qui ne font pas seulement appel à sa raison. 
Quand bien même d'ailleurs cet auditoire serait cultivé et versé dans la chose politique, il ne servirait de rien de chercher à le convaincre par un exposé more geometrico: tant l'objet du discours politique, qui ne saurait être scientifique, que les conditions de son énonciation, devant l'assemblée du peuple, l'interdisent. On s'adressera donc à la phantasia des auditeurs (1404a11), à leur "imagination": il faut comme leur faire voir que ce que l'on dit est bien tel qu'on le dit, et pour ce, parvenir à leur faire entendre ce qu'on ne dit pas. En langage aristotélicien, il faut s'adresser aux auditeurs en produisant devant leurs yeux (poein pro ommatôn) de quoi les convaincre en stimulant leur capacité de représentation et de réflexion. Telles sont les vertus conjuguées de la métaphore et d'autres tours stylistiques.

Voyez d'abord ce qu'il en est de la métaphore. Essentielles aux fables et paraboles, les bonnes métaphores, à distinguer des comparaisons qui, trop longues, ne sont pas à leur place dans le discours politique (Rhét., III, 10, 1410b18-19), sont, dit Aristote, celles qui "font tableau", qui peignent "en produisant devant les yeux", car elles instruisent en signifiant les choses en acte du fait que l'auditeur voit les choses en train de se faire, plutôt que les choses à faire (Rhét., III, 10, 1410b13, b34-35;11, 1411b25). Pour ce faire, il faut qu'elles soient "claires et agréables", mais tout en étant quelque peu "étrangères" sans cesser d'être "convenantes" (III, 2, 1404b4, 1405 a8-b16). Autrement dit, il faut qu'elles soient compréhensibles et euphoniques, mais sans être tirées de trop loin, comme le serait un mauvais jeu de mots, tout en conservant un petit tour énigmatique: la métaphore fera tableau, tout comme les bons jeux de mots, ceux qu' Aristote appelle asteia, "urbains", par opposition aux plaisanteries rustres des paysans (agroika), en instruisant, c'est-à-dire en faisant croire à l'auditeur qu'il a lui-même deviné l'astuce. Afin de rendre l'intelligence de son propos plus prompte et plus aisée (Rhét., III, 11, 1412b21-25), l'orateur jouera des décalages et de l'illusion en télescopant des termes. Il surprendra ainsi l'auditeur en lui faisant croire qu'il a saisi le point par lui-même. L'auditeur se trouve ainsi dans la même situation que le téléspectateur français regardant naguère la célèbre série Les cinq dernières minutes, où le héros, ille Commissaire Bourrel, s'écriait, cinq minutes précisément avant la fin de l'épisode: "Bon sang, mais c'est bien sûr...", et laissait alors aux téléspectateurs le soin de deviner qui était le coupable dans ces fameuses cinq dernières minutes. "Bon sang, mais c'est bien sûr...", voilà en quelque sorte ce que doit pouvoir se dire l'auditeur. Bien faire de bonnes métaphores, qualité que l'on doit à l'exercice de la philosophie, est donc bien tout d'abord la marque d'une personne de qualité, d'un citadin et non d'un paysan.

Mais c'est aussi la marque de quelqu'un qui sait s'y prendre et sait composer son discours en fonction de son auditoire tout en lui masquant les tours auxquels il a recours: l'orateur doit sembler parler "par nature" et non "par art" (Rhét., III, 2, 1404b18-26, b36; 7, 1408 b5-6; 16, 1417b8) et pour cela chercher à déjouer les illusions ou cruelles vérités que pourrait engendrer son discours. D'où l'attention que cet orateur s'efforçant de convaincre en paraissant vertueux portera au paralogisme et au rythme. Au paralogisme tout d'abord. Bien que répréhensible en saine logique, car la réfutation n'y est qu'apparente, l'orateur pourra y avoir recours si le style qu'il emploie alors est "approprié, convenant": on conclut paralogistiquement à la véracité de l'orateur s'il a parlé pathétiquement de choses pathétiques (III, 7, 1408a10-25). Le choix des termes appropriés distingue ainsi l'homme cultivé du 
rustre (1408a32). Mais il y a plus: le paralogisme convient particulièrement au style oral. Apparenté au procédé de l'amplification par l'usage des répétitions et des asyndètes, le paralogisme fait croire qu'il y a plus dans ce qui est dit que ce qui est dit réellement (III, 12, 1413b32, 1414a5). L'amplification tient en effet à la suppression des conjonctions: en désunissant ce que la conjonction a pour fonction d'unir, la suppression des conjonctions donne à entendre une multiplicité en lieu et place d'une seule et même chose. Paradoxalement, l'usage des répétitions par suppression des conjonctions produit l'impression de brièveté recherchée: conjoindre, c'est démontrer, au risque de perdre son auditoire en route; disjoindre sans se priver de répéter, c'est marteler afin de convaincre.

De ces considérations, et je finirai par là, relèvent également ce que dit Aristote du rythme (Rhét., III, 8) et du style "moderne" (III, 9). La composition du discours doit en effet relever du style "implexe" (katestramenê) et non du style "coordonné" (eiromenê), ce dernier étant l'ancien style dont la caractéristique était de donner au discours son unité grâce aux conjonctions. Ce qui le rendait désagréable (aêdês) à entendre du fait qu'on ne pouvait pressentir la fin du discours, une conjonction pouvant toujours venir s'ajouter à une autre conjonction. C'est pourquoi Aristote qualifie le style de ce type de discours d' «illimité" (III, 9, 1409a24-36). A l'inverse, le style implexe, c'est-à-dire procédant par périodes, répond aux mêmes nécessités que le rythme: fuir l'indéterminé ou illimité, désagréable parce que non instructif, en recherchant le nombre (III, 8, 1408b27; 9, 1409b5-6). Dès lors le péan sera le rythme idéal puisque, non métrique, il ne saurait se transformer en vers et se dissimulera donc le plus aisément (malista lanthanein, 1409a9). Bref, le péan est le bon rythme car, hommage anticipé à la très belle Annie Lennox, il est "eurythmique".

L'eurythmie renvoie à la "bonne forme", à la "forme harmonieuse", laquelle, à en croire Platon, devrait s'obtenir uniquement à partir des proportions réelles, mais qui s'obtient bien plus sûrement à partir des proportions apparentes dès lors qu'on tient compte du point de vue des spectateurs, ce qu'instaurèrent les artistes de la seconde moitié du IVè siècle av. J.-C. Aussi, si l'on se souvient que le discours, notamment politique, s'adresse à la phantasia des auditeurs, il est tentant de comprendre qu'Aristote demande à l'orateur de procéder comme l'architecte donnant à voir une belle forme pour la phantasia (to pros phantasian eurythmon) en recherchant l'égalité et l'eurythmie relatives à la vue (pros opsin) et non à la réalité ou à la vérité (kat'alêtheian), ainsi qu'il est dit dans ce texte anonyme intitulé ti to skênographikon. Dernier argument: puisque le nombre non métrique rend la période plaisante, instructive et facile à comprendre, l'auditeur croit (oietai, 1409b3) toujours posséder la chose conclue car il peut la prévoir. En témoigne la période composée de membres antithétiques: elle est agréable parce qu'elle ressemble à un syllogisme réfutatif (eoike, $1410 \mathrm{a} 22$ ), or on se souvient que celui-ci a les faveurs du public en ce que, brillant et aisé à suivre, il donne à l'auditeur le sentiment d'être rehaussé.

\section{Conclusion}

Pro ommatôn poein, fabriquer devant les yeux, oreilles en l'occurrence, d'où cet examen des procédés stylistiques appropriés au discours oral, pro ommatôn poein, donc, 

dans la Rhétorique d'Aristote.

semble bien renvoyer à ce travail pros phantasian, "relatif à l'«imagination", Il est en ce sens hautement significatif que la très grande majorité des exemples de métaphores "faisant tableau" soit tirée de discours politiques (III, 10, 1411a1-b21). Le vertueux, s'il entend conseiller efficacement, doit donc se donner l'apparence d'être tel, c'est-à-dire de bon conseil. Mais il ne le pourra que par la force de son seul discours, ce à quoi la philosophie l'aura entraîné en lui apprenant à "bien métaphoriser". Il n'y aura là pourtant nulle tromperie puisqu'il s'agira seulement de donner des arguments proprement audibles et de se faire paraître pour ce que l'on est en "se mettant en scène". Au risque bien évidemment d'avoir affaire à un aigrefin, mais c'est une autre histoire...et une question de métier: on n'a jamais que les orateurs qu'on mérite!

\section{Note}

1 - Cf. Rhét., I, 2, 1357a1, 11; II, 21, 1395b1; 22, 1395b23; III, 1, 1403b11, 22, 36; 7, 1408a30; 14, 1415a34-17.

LABARRIÈRE, J.-L. Fables and metaphors as logical proofs in the political speech in Aristotle's Rhetoric. Classica, São Paulo, 13/14, p. 233-240, 2000/2001.

RÉSUMÉ: Aristote considère que, au sein des preuves morales, la preuve par le caractère est la plus adaptée au discours politique tandis que celle cherchant à exciter les passions des auditeurs est plus adaptée au discours judiciaire. Cette preuve (pistis), qui a pour fin de faire paraître l'orateur d'une certaine qualité, rend la harangue plus noble que le plaidoyer. Mais en ce qui concerne les preuves logiques, le plaidoyer a l'avantage du fait que, portant sur le passé, il peut user d'enthymèmes. La harangue, portant sur l'avenir, use des exemples (paradeigmata), qui sont de deux types: les exemples historiques, qui devraient être les plus forts, ou les fables et paraboles, auxquelles l'orateur a recours car l'histoire est souvent muette. C'est cette utilisation des fables qui est ici interrogée: il ne faut en user que si l'on sait apercevoir les ressemblances, trait qui caractérise celui qui sait faire des métaphores.

MOTS-CLÉS: Aristote; rhétorique; discours politique; preuves; métaphores. 